\title{
Combined use of digital imaging technologies: ortho-surgical treatment
}

\author{
L. Petitpas
}

Private practice, 54700 Pont-à-Mousson

Are digital dental impressions able to replace traditional dental impressions? This question haunts many orthodontists who are considering investing in intraoral scanners.

In my opinion, replacing the conventional procedure for taking impressions is not necessarily the most important objective.

In fact, these devices bring a much greater value to our treatments by optimizing and controlling our mechanics, while serving as a precise means of planning, previewing, and evaluating our results.

The presentation of the following complex case shows how the combination of different digital imaging technologies can be useful in difficult cases such as surgical cases.

This patient was seen for temporomandibular joint (TMJ) pain combined with limited mouth opening.

Clinical examination revealed a class II skeletal malocclusion with a very convex hyper-divergent profile combined with a mandibular lateral deviation on the right.

Cephalometric profile analysis shows maxillary proalveolia and skeletal class II. Facial cephalometric analysis revealed a convergence of gonic and occlusal planes, confirming mandibular deviation.

At this point, digital imprinting (3M True Definition Scanner) is used to refine the diagnosis, quantify asymmetries, plan treatment, and visualize objectives.

An earlier magnetic resonance imaging of the TMJ confirms what the clinical examination suggested: a bilateral irreducible meniscal dislocation.
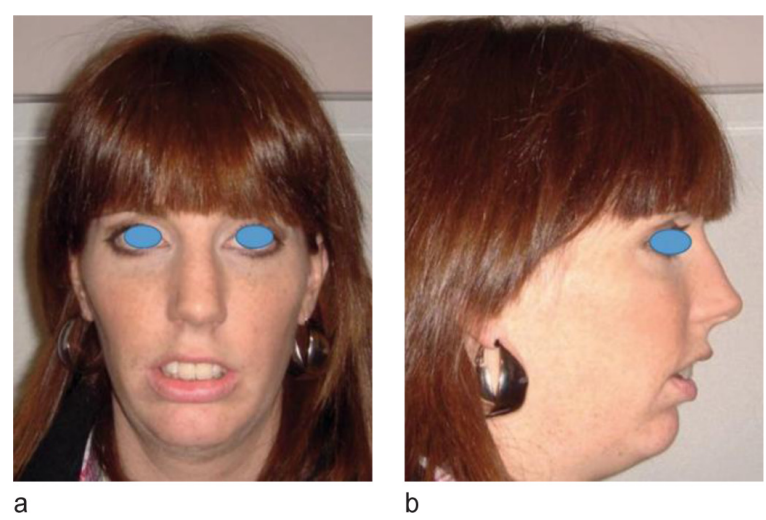

Figure 1

a) Extraoral picture of the face before treatment. Mandibular lateral deviation on the right, and resting labial incompetence. b) Extraoral profile picture. Significant convexity and retrognathia.

\section{Address for correspondence:}

Laurent Petitpas - 35c impasse des Brasseries - 54700 Pont-à-Mousson

This is an Open Access article distributed under the terms of the Creative Commons Attribution License (http://creativecommons.org/licenses/by/4.0), which permits unrestricted use, distribution, and reproduction in any medium, 


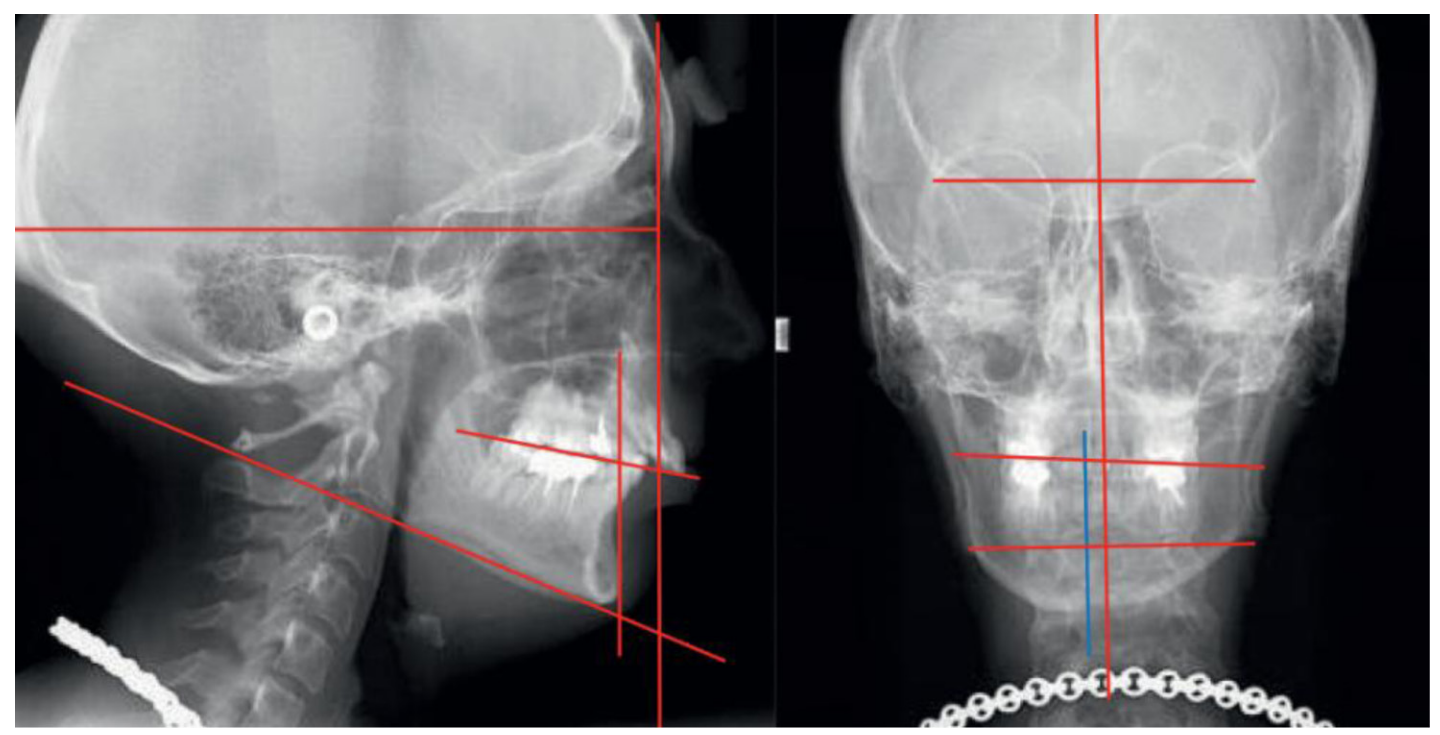

Figure 2

Frontal and profile teleradiography before treatment.

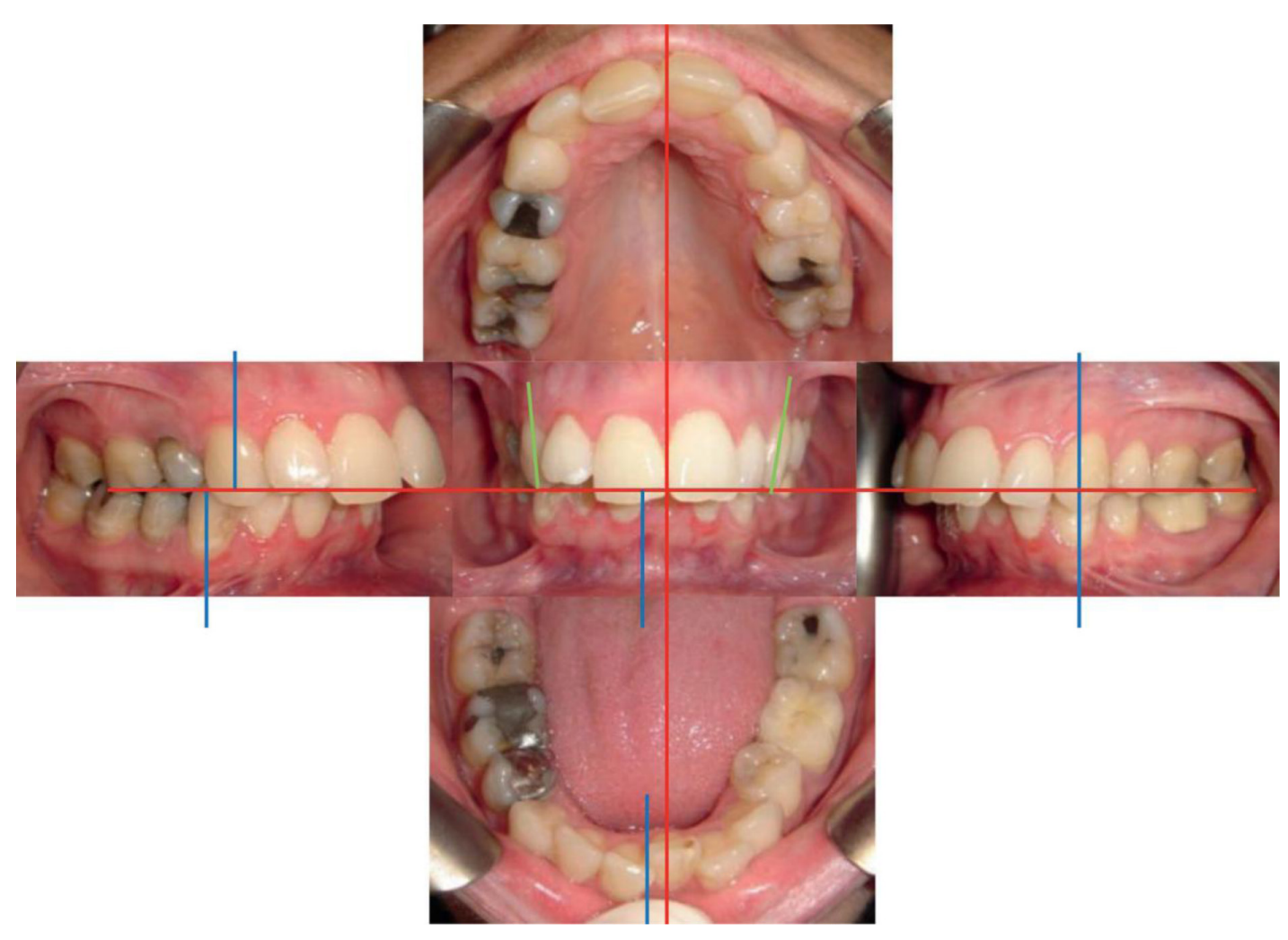

Figure 3

Intraoral photos before treatment: asymmetric class II malocclusion, proalveolia, increased overbite, and deviation of mandibular incisor medium on the right. 


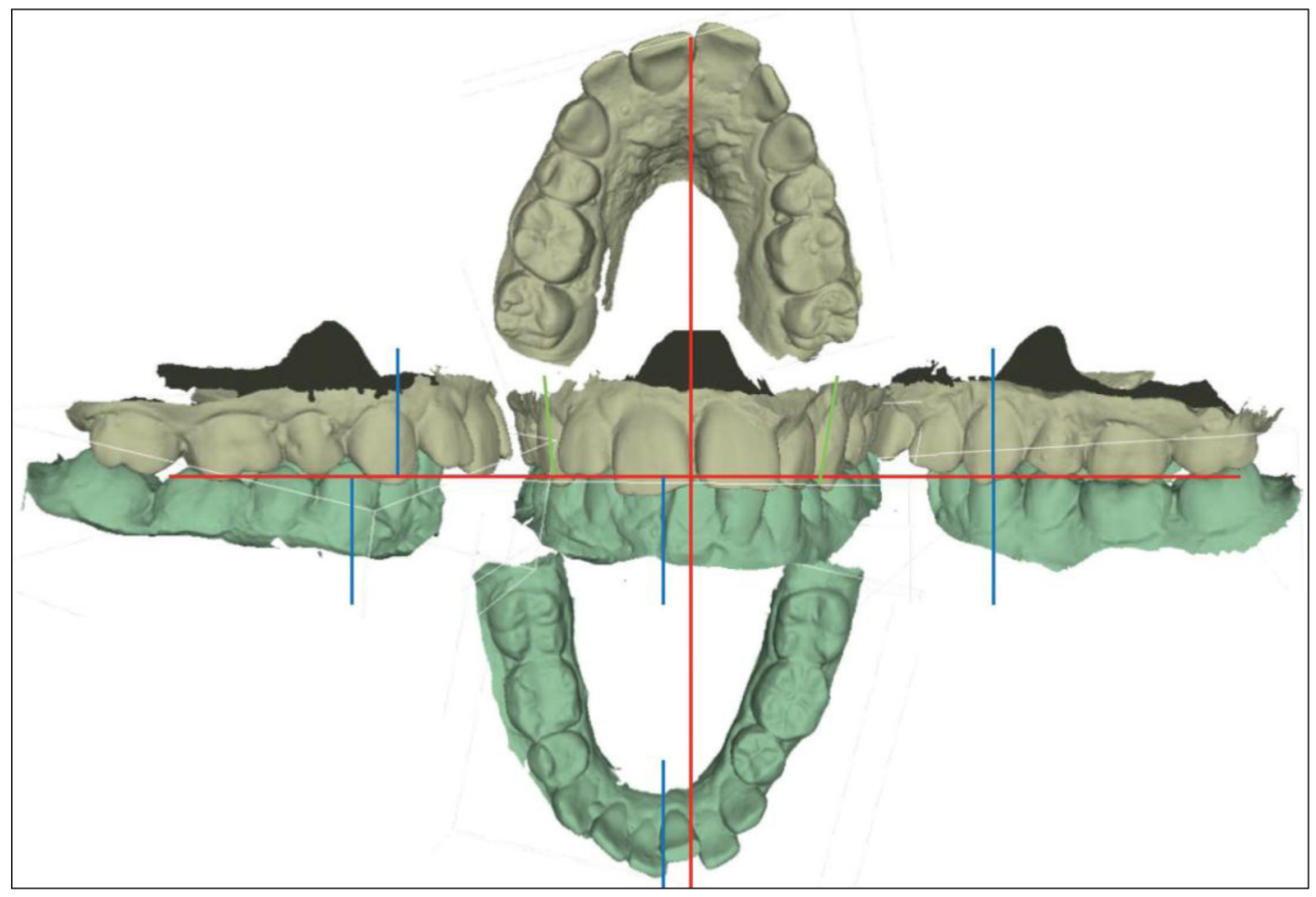

Figure 4

Asymmetry and torsion of the occlusal plane are highlighted on digital models with much more accuracy than on plaster models.
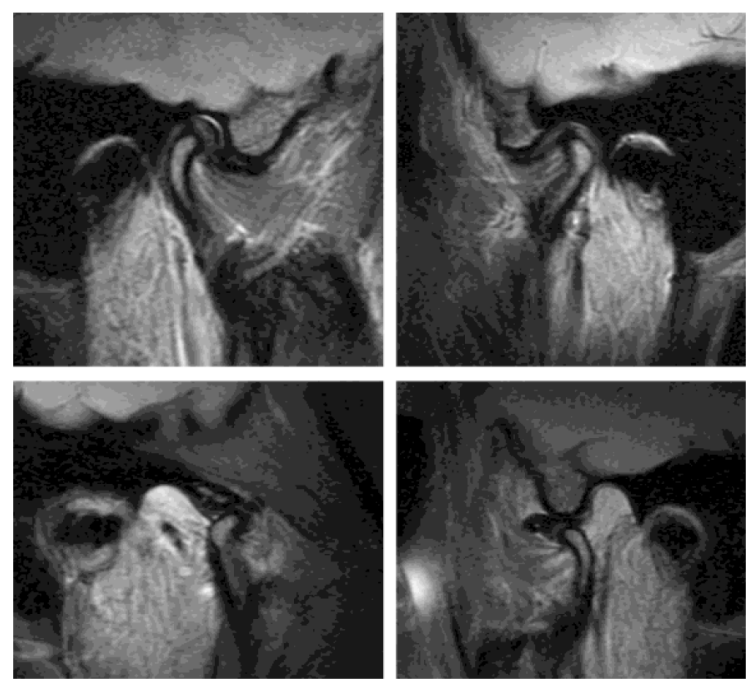

Figure 5

Magnetic resonance imaging of the TMJ: Bilateral irreducible meniscal dislocations causing pain and limiting mouth opening. 


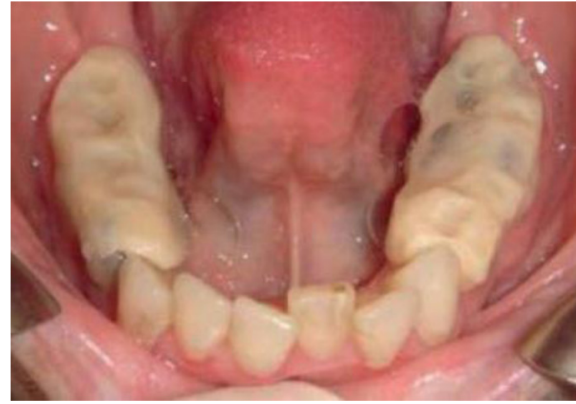

Figure 6

Repositioning gutter.

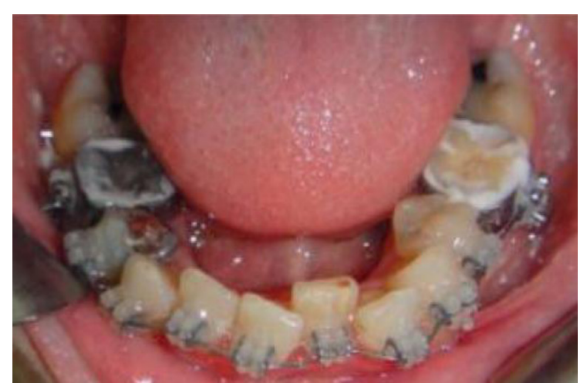

Figure 8

Vestibular multi-attachment to the mandibular arch.

The chosen treatment plan involves a surgical-orthodontic protocol, which begins with the wearing of a mandibular joint orthopedic repositioning gutter to provide patient relief.

The orthodontic preparation is then performed using a fixed, vestibular mandibular, and maxillary lingual technique. The digital impressions having been used again to create the lingual apparatus (Incognito Lingual Appliance System).

In addition to decompensating the malpositions due to lateral deviation, the orthodontic preparation made it possible to reposition the mandible and restore asymptomatic joint conditions.

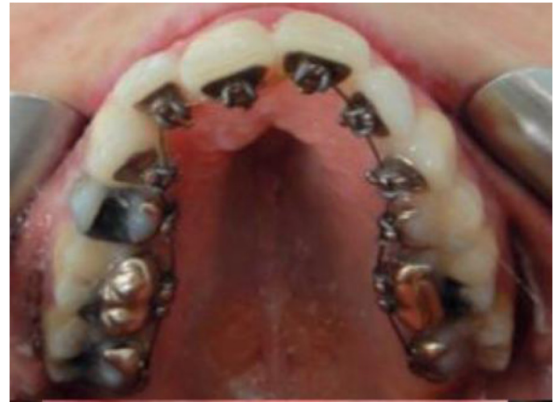

Figure 7

Lingual multi-attachment to the maxillary arch.

Class II intermaxillary elastics were used to maintain asymmetric joint positioning during this treatment step.

Complete assessment with a digital impression was carried out at the end of the 14-month orthodontic preparation to objectify the results of the orthodontic preparation. Significant improvement was observed. The maxillary arch is now symmetrical, the canine axes corrected, and the Wilson curve balanced.

Moreover, TMJs are now asymptomatic and function properly.

The superimposition of the digital data allows both the visualization of the orthodontically obtained modifications and previsualization of the surgical orthopedic repositioning.

The surgical procedure is planned by combining digital impressions and CBCT scans, thus making it possible to perform a bone set-up.

The surgery is then performed according to the treatment plan.

At the end of the surgery, 4 months of orthodontic finishing allowed the treatment to be completed. A new complete assessment is then conducted to compare the results. 


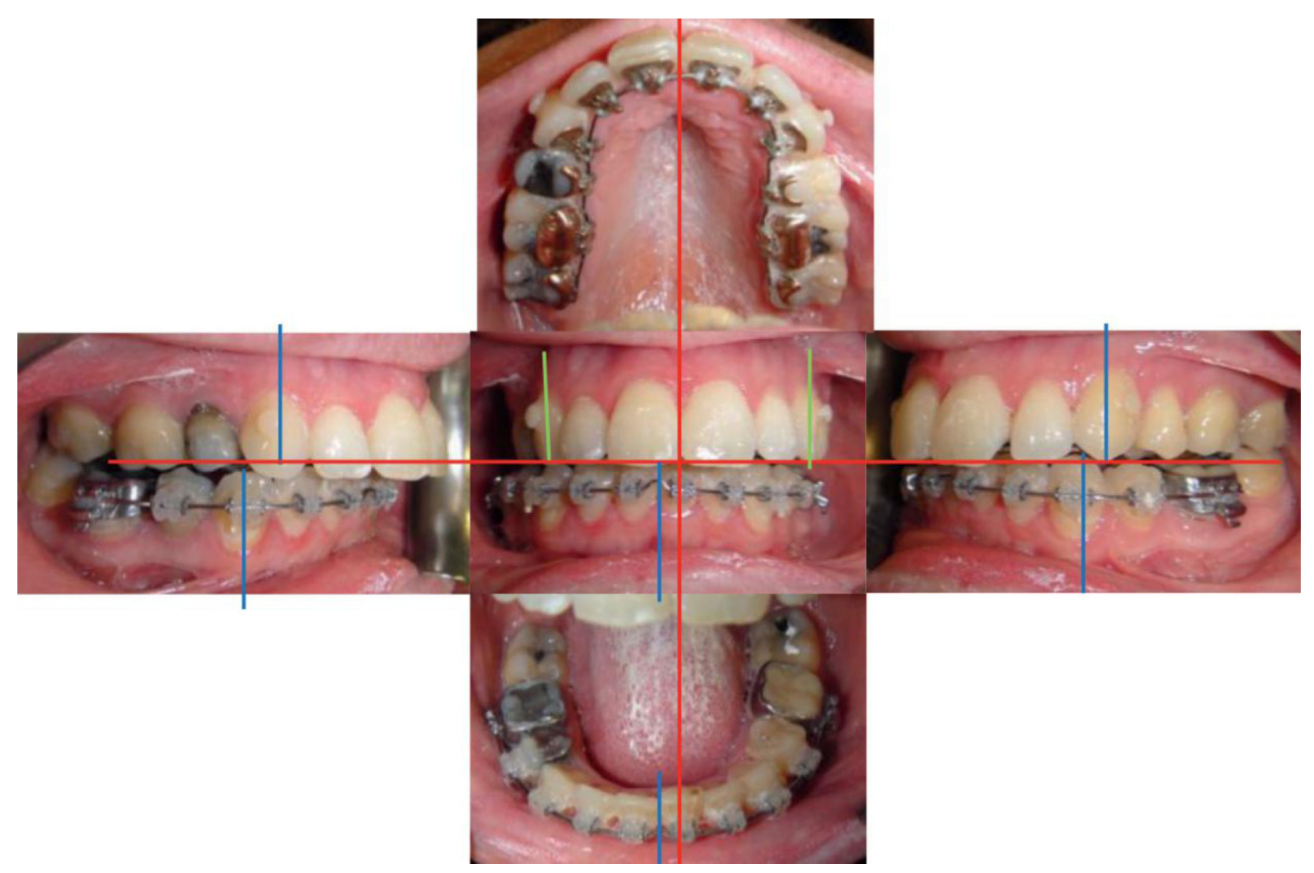

Figure 9

Intraoral pictures after orthodontic preparation.

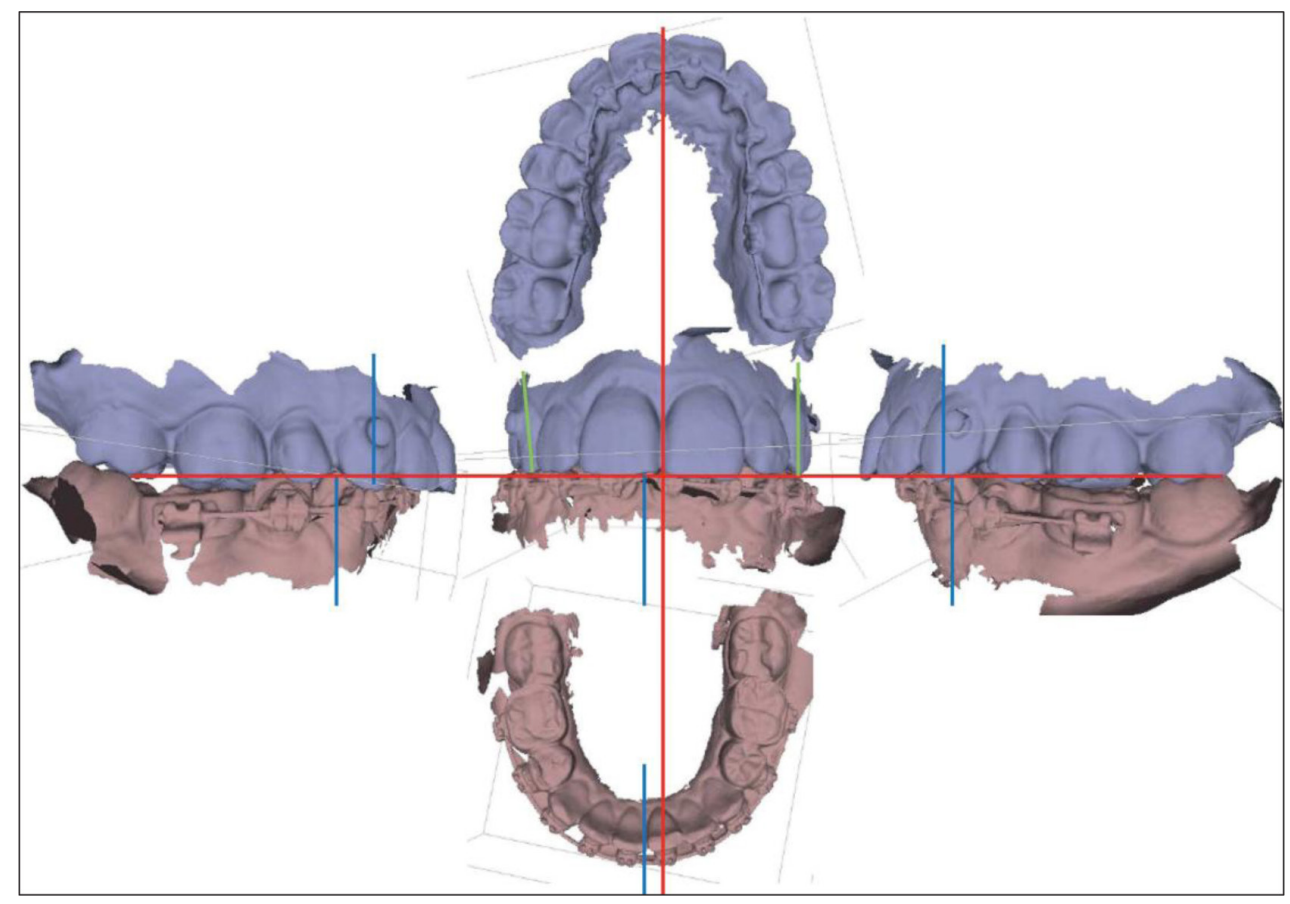

Figure 10

3D digital models after orthodontic preparation. 

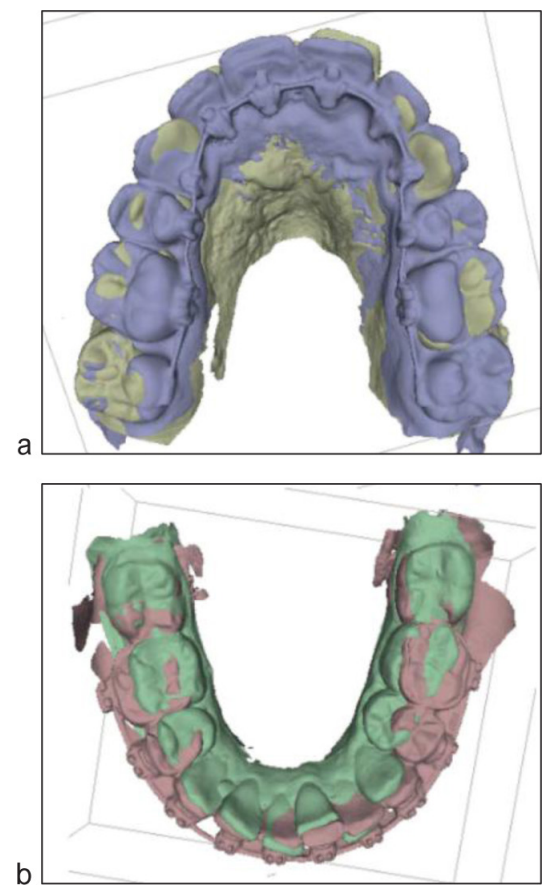

Figure 11

a) Digital superimposition before treatment and after orthodontic preparation of the maxilla.

b) Digital superimposition before treatment and after mandibular orthodontic preparation.

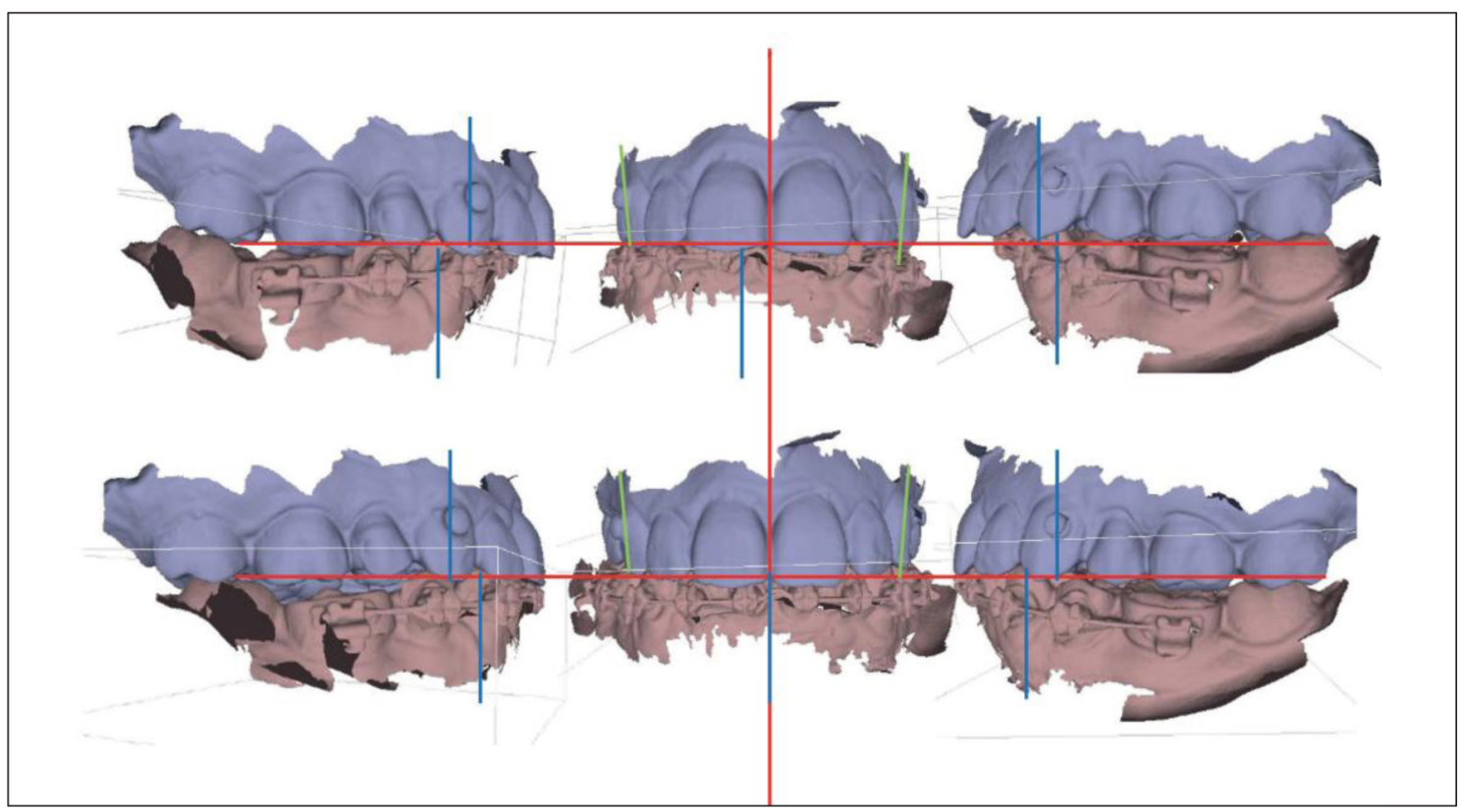

Figure 12

Digital simulation of the surgical effects with simulation of post-surgical occlusion: restoration of class I occlusion, evaluation of expansion, and correction of asymmetry. 


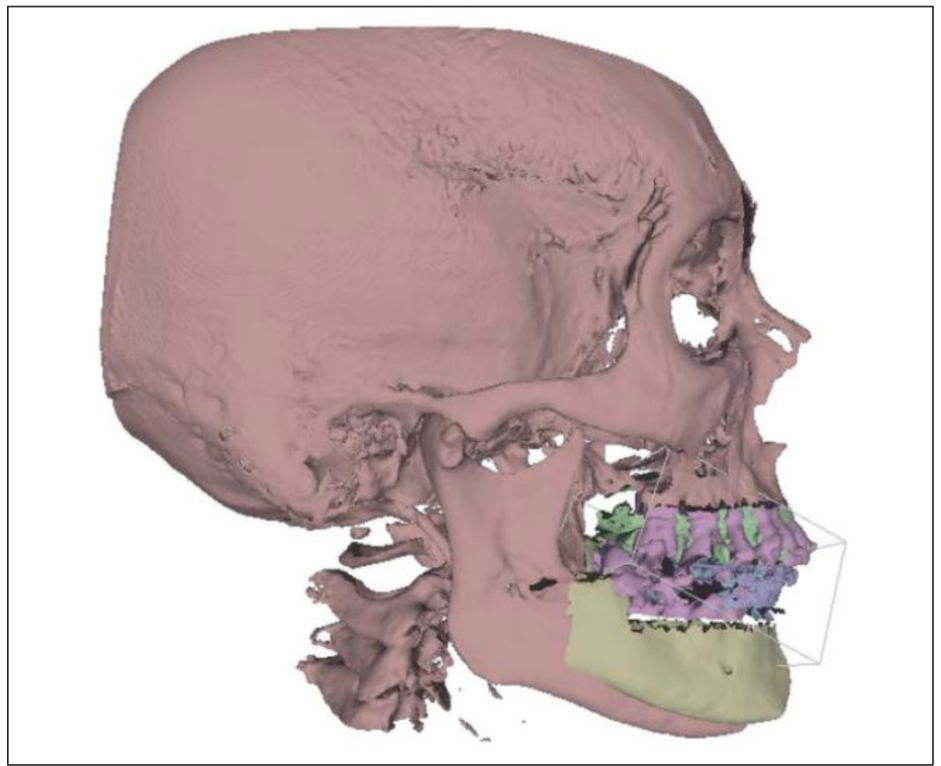

Figure 13

Simulation of surgery from CBCT and digital impressions.

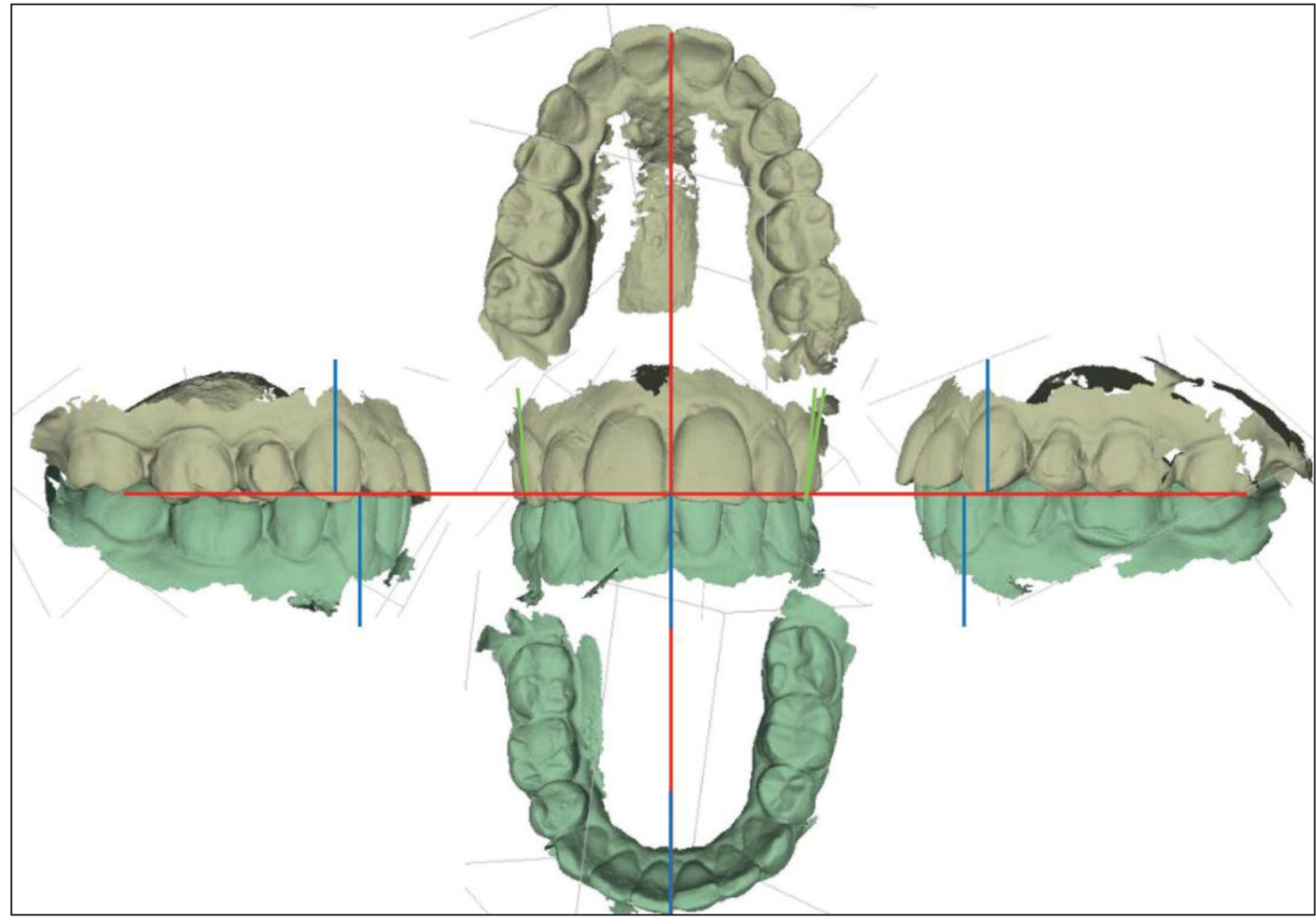

Figure 14

Digital models after treatment. 


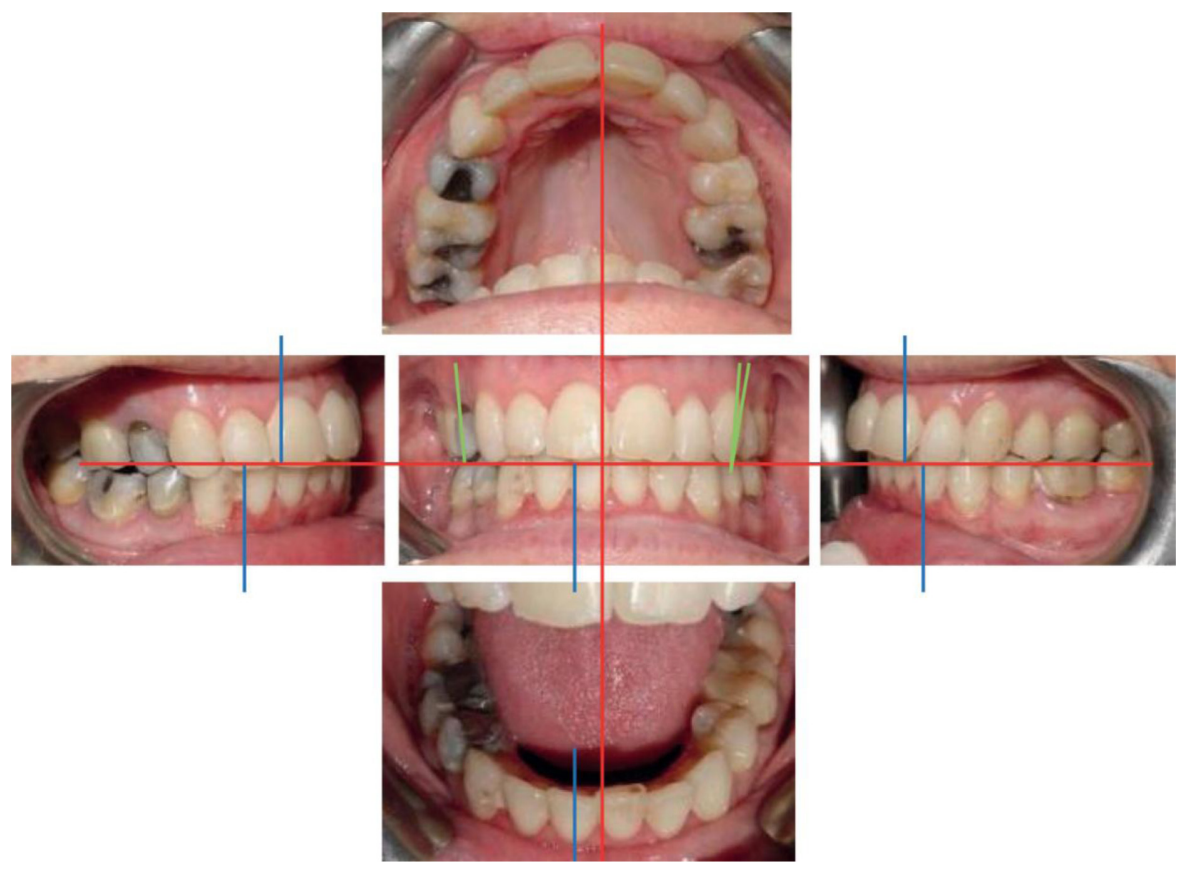

Figure 15

Intraoral pictures after treatment. Centered medians; bilateral dental class I obtained.

Comparison with the initial markers at the start of treatment (see Figure 9).
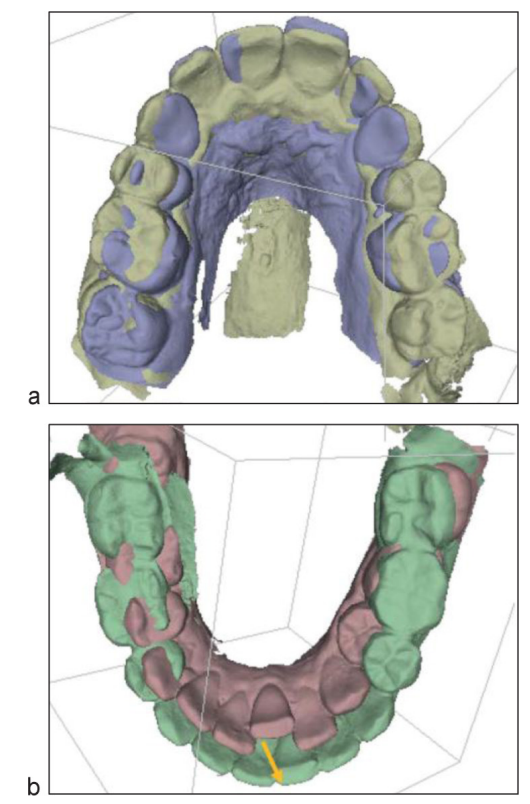

Figure 16

a) Superposition before and after maxillary treatment. b) Superposition before and after mandibular treatment. The effects of the surgery are clearly visible: 6-mm advancement and 3-mm rotation. 


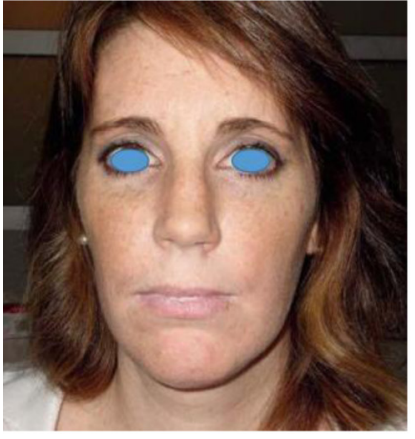

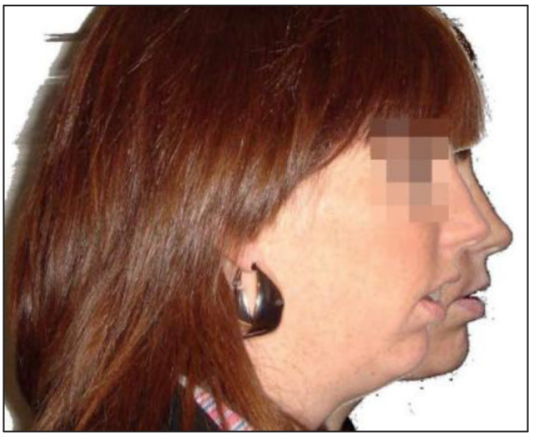

b

Figure 17

a) Extraoral picture of the face after surgical treatment. b) Profile evolution by looking at preand post-surgical photographs.

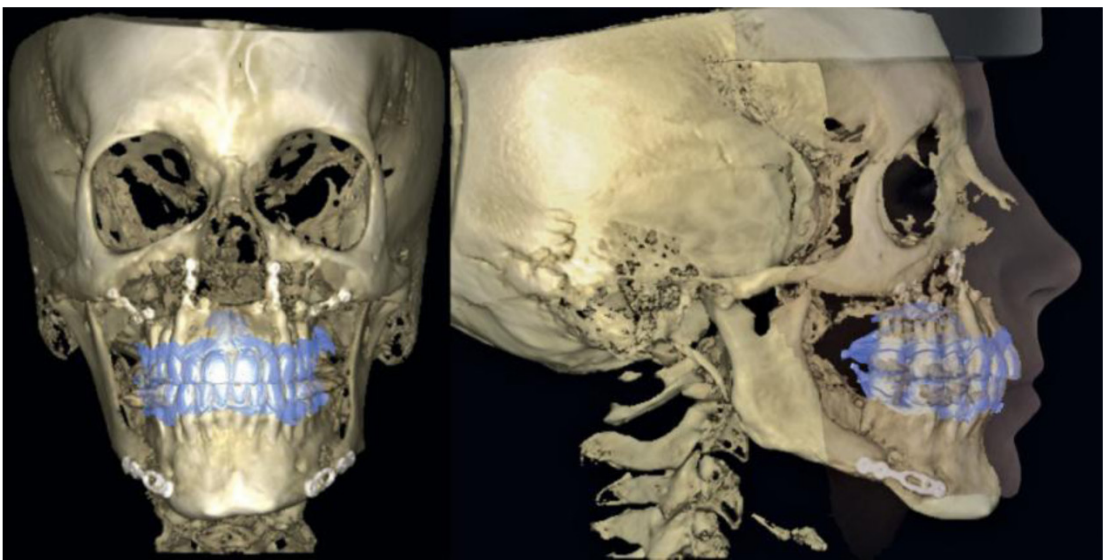

Figure 18

Cranial CBCT combined with digital end-of-treatment models. In the right lateral view, the patient's skin profile is visible, making it possible to check the coordination between the bone bases and soft tissues.

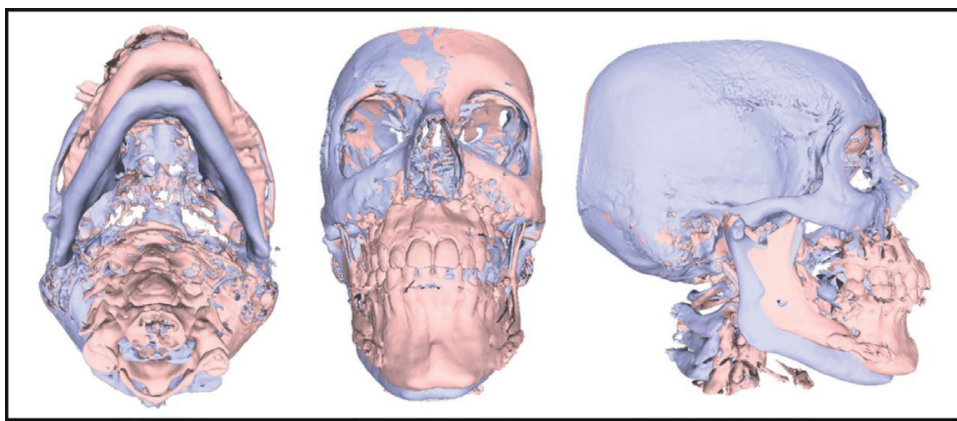

Figure 19

Basal, frontal, and lateral views of CBCT superimpositions before and after treatment. 
Additional assessments were performed by combining CBCT with digital models and by superimposing CBCTs before and after surgery.
The effects of the treatment are visible and the changes in the mandible are notable.

Finally, TMJ repositioning was verified.

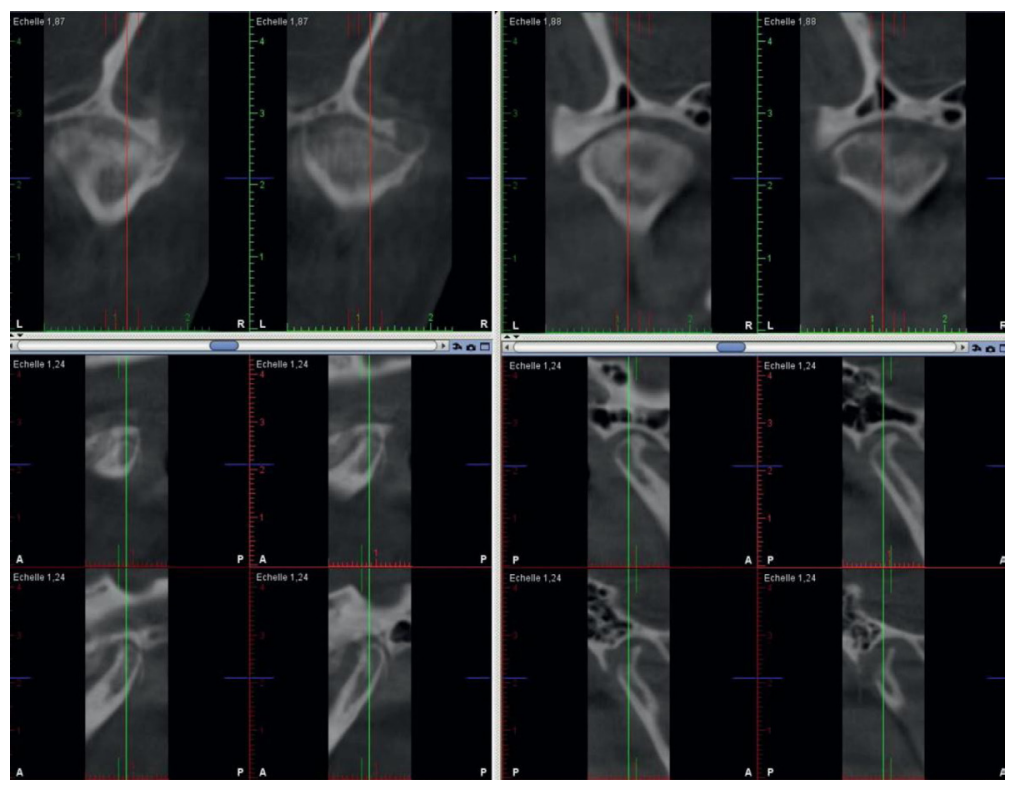

Figure 20

TMJ visualization on cone beam section.

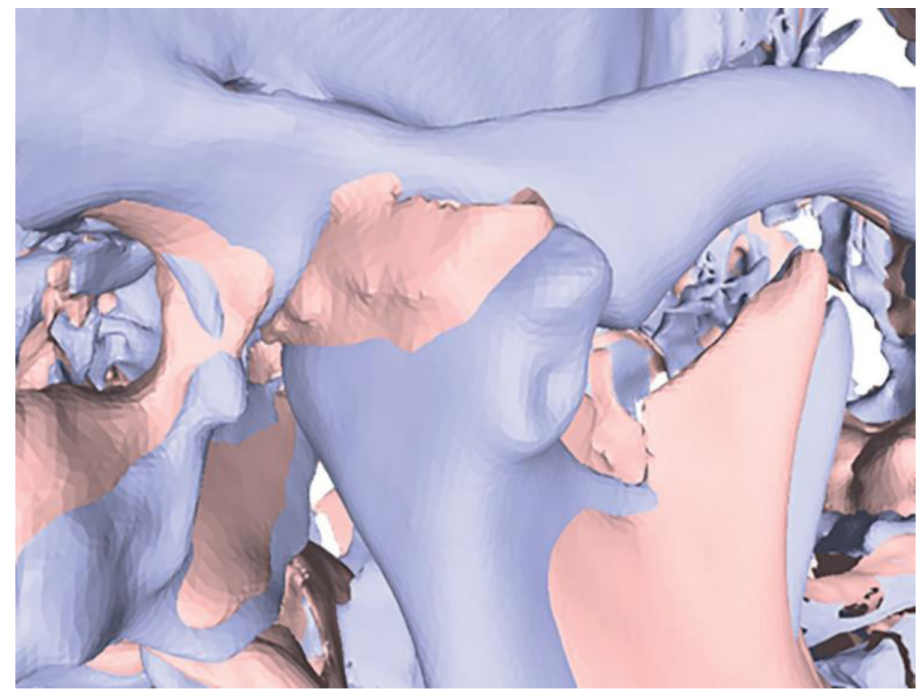

Figure 21

Superimposition of CBCT centered on the TMJ area showing recentering. 
The comparison of the two 3D examinations shows that the center of the lower lip has changed significantly and moved $13 \mathrm{~mm}$ above and forward with chin alignment.

The finishes were made from digital impressions. The compression gutters were made directly at the practice on models made of polylactic acid (PLA) using a 3D printer.

This clinical case perfectly illustrates the new opportunities offered by the use of digital techniques.
They are a valuable aid for diagnosis, planning, device design, and evaluation of the different treatment stages.

While there are advantages to the individual use of each technique, it is undeniable that their combined use maximizes the benefits that can be derived from it. The fused modeling of teeth, bone bases, and soft tissues results in the creation of a virtual patient.

This approach makes it possible to visualize the treatment plan at any level, both dental and cutaneous.

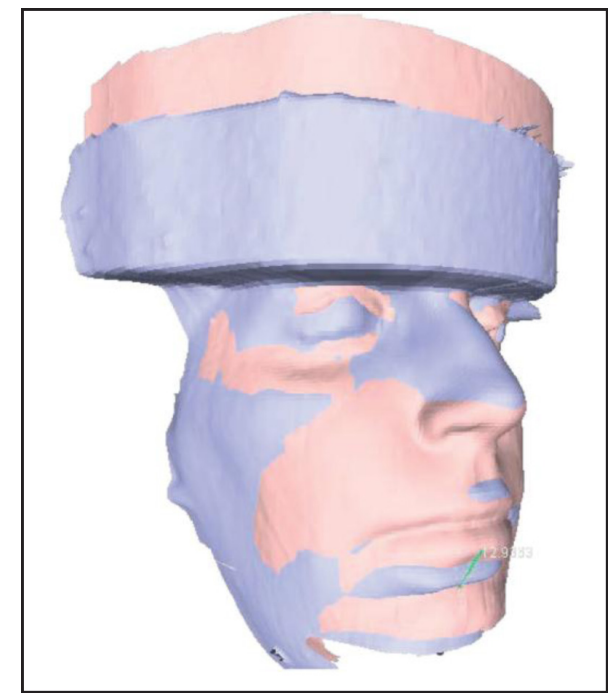

Figure 22

Facial changes, especially at the level of the lips. 3D modeling allows better evaluation than $2 D$.

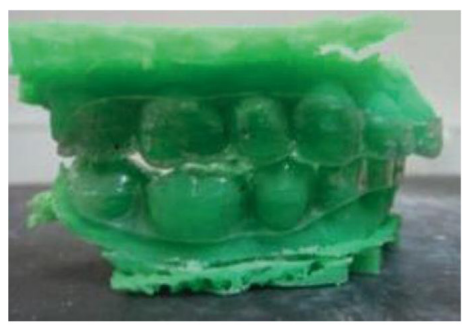

a

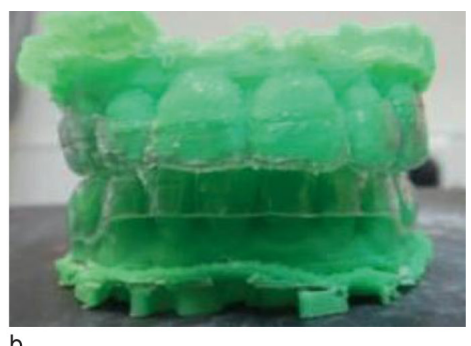

b

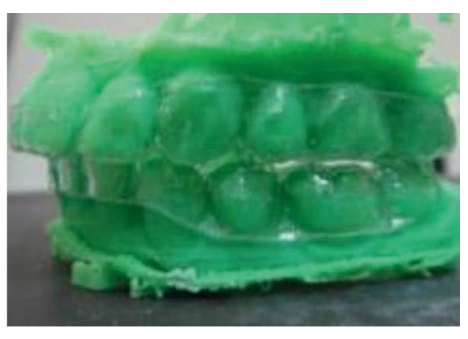

C

Figure 23

Thermoformed gutters on PLA models. 


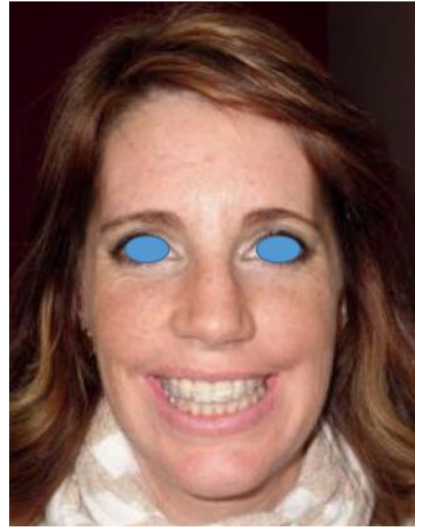

Figure 24

Extraoral photo of the face during the period of contention showing balanced esthetics.
For the orthodontist, it facilitates the early detection of possible treatment plan errors while promoting communication with patients, which is the key to therapeutic success. In the long term, this wealth of information could lead to a better understanding of therapeutic mechanisms and their success.

Conflict of interest: The authors declare no conflicts of interest. 